Universitaria Friuli Centrale, Rheumatology Clinic, Udine, Italy; ${ }^{3}$ University of Udine, Department of Medical and Biological Sciences, Udine, Italy; ${ }^{4}$ University of Trieste, Department of Medicine, Surgery and Health Sciences, Trieste, Italy

Background: Tenosynovitis can occur in patients with Juvenile Idiopathic Arthritis (JIA) and may be clinically difficult to distinguish from joint synovitis. The role of musculoskeletal ultrasound (msk-US) is still discussed in the management of JIA but recent studies supported the utility of msk-US especially in detection of tenosynovitis. There is no consensus treatment for tenosynovitis in children with JIA and almost all studies focused on tendon sheath injection with glucocorticoids. Objectives: The aims of the study were: i) to define the prevalence of tenosynovitis of the ankle in JIA patients and ankle swelling; ii) to describe the clinical characteristics of patient with tenosynovitis and to analyze different response to treatment.

Methods: We conducted an observational cross-sectional study of a group of patients with JIA followed at the Rheumatology Service of the Maternal and Child Institute "Burlo Garofolo" of Trieste. We enrolled all the patients who reported a swelling of the ankle at least once during the follow-up period and, among these patients, we included only those who underwent msk-US. Based on both clinical and sonographic examination, we identified patients with tenosynovitis and we described their demographical and clinical characteristics as well as the therapeutic approach undertaken in this group of patients.

Results: On December 31st 2019, 56 swollen ankles of 48 patients were assessed with msk-US: 22 ankles showed sonographic signs of synovitis (39\%), 16 ankles of both synovitis and tenosynovitis (28\%), 14 ankles of tenosynovitis only (25\%). Overall, tenosynovitis was detected on twenty-seven $(56 \%)$ out of 48 children with at least a swollen ankle. The majority of patient were females (70\%) and the most affected tendon was the tibialis posterior (66\%). Twenty-five patients with tenosynovitis (92\%) achieved clinical and radiological remission: seven out of 26 patients $(26 \%)$ treated with methotrexate achieved clinical and radiological remission without the addition of other therapies; fifteen out of seventeen patients $(88 \%)$ treated with a biological drug responded to the therapy, of which eleven $(73 \%)$ were in combination therapy with methotrexate.

Conclusion: We observed that more than $50 \%$ of the patients with a swelling of the ankle presented a tenosynovitis and among these patients about $50 \%$ did not show sonographic sign of synovitis. Msk-US was decisive in order to identify tenosynovitis and to characterize ankle swelling in JIA patients. Among patients with tenosynovitis biological therapy alone or in association with immunomodulating therapy showed effectiveness in inducing disease remission.

REFERENCES

[1] Peters SE, Laxer RM et al. Ultrasound-guided steroid tendon sheath injections in juvenile idiopathic arthritis: a 10-year single-center retrospective study. Pediatr Rheumatol Online J. 2017 Apr 11;15(1):22.

[2] Lanni S, Bovis F et al. Delineating the Application of Ultrasound in Detecting Synovial Abnormalities of the Subtalar Joint in Juvenile Idiopathic Arthritis. Arthritis Care Res (Hoboken). 2016 Sep;68(9):1346-53.

[3] Cimaz R, Giani T et al. What is the real role of ultrasound in the management of juvenile idiopathic arthritis? Ann Rheum Dis. 2020 Apr;79(4):437-439. doi: 10.1136/annrheumdis-2019-216358

[4] Lanni S, Marafon DP et al. Comparison between clinical and ultrasound assessment of the ankle region in juvenile idiopathic arthritis. Arthritis Care Res (Hoboken). 2020 Apr 27.

[5] Laurell L, Court-Payen M et al. Ultrasonography and color Doppler in juvenile idiopathic arthritis: diagnosis and follow-up of ultrasound-guided steroid injection in the ankle region. A descriptive interventional study. Pediatr Rheumatol Online J. 2011 Jan 29;9(1):4.

Disclosure of Interests: None declared

DOI: 10.1136/annrheumdis-2021-eular.4272

\section{POS1334 RHEUMATOLOGY PATIENT ATTENDANCE TO THE PAEDIATRIC EMERGENCY DEPARTMENT}

M. Al-Attar ${ }^{1} .{ }^{1}$ Paediatric Emergency Department, Royal Manchester Children's Hospital, Manchester, United Kingdom

Background: A large proportion of patients present to the paediatric emergency department (PED) with non-urgent problems [1]. With growing pressures on services, there is a need to reduce unnecessary attendances. Children with rheumatological conditions may attend PED with flares of their condition or unrelated presentations. These patients may be immunosuppressed due to steroid, disease-modifying or biologic therapy; PED attendance may therefore pose a serious infection risk.

Objectives: To evaluate why children with rheumatological conditions attend the PED and whether these attendances are avoidable.

Methods: This study was run in Royal Manchester Children's Hospital, a UK hospital with a PED and tertiary paediatric rheumatology services. Patients under rheumatology who attend PED are usually seen directly. A dataset of all patients referred directly to paediatric rheumatology between 01/01/19 and 31/12/19 was obtained from electronic records $(n=59)$. The age, sex and reason for attendence were collected, as well whether they were admitted/discharged. Further data on management was collected from discharge letters for those with joint pain/swelling.

Results: This cohort had 36 females and 23 males, with an average age of 11.9 years (range 1-18). 28 were discharged (47.5\%) and 30 were admitted $(50.8 \%)$ One patient left before being seen. The average waiting time was 3.97 hours. Table 1 summarises reasons for attendance to PED and final outcome. The most common reason for attendance was joint pain/swelling $(n=14,23.7 \%)$. Of these, nine were discharged with advice on analgesia or steroid dosing and for early review in clinic. There was no documentation on whether there had been any attempt to access the available rheumatology helpline prior to attending PED. Non-rheumatology-related reasons for attendance are also shown in Table 1, and notably include 20 patients with infections, $75 \%$ of whom were admitted to hos pital. Chicken pox was the second most common reason for attendance $(n=5$ $8.5 \%)$; all patients attending with chicken pox or shingles $(n=6)$ were admitted.

Table 1. Reasons for attendance to PED and final outcome

\begin{tabular}{lcc}
\hline Reason for attendance & Number admitted & Number discharged \\
\hline Joint pain/swelling & 5 & 9 \\
Chicken pox & 5 & 0 \\
Viral URTI & 1 & 4 \\
Rash & 0 & 4 \\
Pneumonia & 3 & 0 \\
SLE flare & 3 & 0 \\
Influenza A & 2 & 0 \\
GPA flare & 2 & 0 \\
Asked to attend due to blood results from clinic & 2 & 0 \\
Drug reaction & 0 & 2 \\
Minor injury & 0 & 2 \\
Shingles & 1 & 0 \\
Oral herpes simplex & 1 & 1 \\
Orbital inflammation & 1 & 0 \\
Urinary tract infection & 1 & 0 \\
Dysphagia & 1 & 0 \\
Gastroenteritis & 1 & 0 \\
Cerebral infarcts & 1 & 0 \\
Constipation & 0 & 1 \\
Chest pain & 0 & 1 \\
Abdominal pain & 0 & 1 \\
Enlarged lymph node & 0 & 1 \\
Picking up prescription & 0 & 1 \\
Unresponsive episode & 0 & 1 \\
\hline
\end{tabular}

Conclusion: Our study shows that attendance to PED with joint pain/swelling is usually avoidable in patients known to paediatric rheumatology services. Appropriate alternative services are needed to reduce hospital attendances; nurse-led helplines are beneficial, but still face some challenges in both availability and accessibility [2]. It is also important that patients and parents are given action plans for acute flares, which would ideally involve early clinic review rather than PED attendance. Our data also showed that patients are spending a long time waiting in PED, which needs to be avoided particularly in the context of immunosuppression. This is especially poignant now in light of the Covid-19 pandemic Direct referral to rheumatology with non-rheumatological problems likely reflects a desire for specialist advice in the context of complex conditions and medications, for example with regards to infections whilst on immunosuppressive medications, or uncertainty about whether new symptoms are related to the underlying condition. Expansion of helpline services and new approaches such as remote consultations should be explored.

\section{REFERENCES:}

[1] Poku BA et al. Reducing repeat paediatric emergency department attendance for non-urgent care: a systematic review of the effectiveness of interventions. Emerg Med J. 2019 Jul;36(7):435-442.

[2] Palmer et al. Rheumatology helpline: challenges in the biologic therapy era Br. J. Nurs. 2011 20:12, 728-732

Disclosure of Interests: None declared

DOI: 10.1136/annrheumdis-2021-eular.4325

\section{Other orphan diseases}

\section{POS1335 CLINICAL CHARACTERISTICS OF THE PULMONARY ARTERY INVOLVEMENT IN PATIENTS WITH BEHÇET'S SYNDROME: A SINGLE-CENTRE EXPERIENCE OF 61 PATIENTS}

D. Sahin Eroglu ${ }^{1}$, M. Torgutalp ${ }^{1}$, S. Baysal ${ }^{2}$, A. Colaklar ${ }^{3}$, S. Sezer ${ }^{1}$, M. E. Yayla ${ }^{1}$, C. Uzun ${ }^{3}$, T. M. Turgay ${ }^{1}$, G. Kınıklı ${ }^{1}$, A. Ates ${ }^{1} .{ }^{1}$ Ankara University Faculty of Medicine, Department of Internal Medicine, Division of 